\title{
Recomendaciones para contratar servicios en la 'nube'
}

\author{
How to Hire the Cloud Computing Services' Advices \\ Recomendações para contratar serviços em 'nuvem'
}

\section{Resumen}

La Computación en la Nube, nueva tecnología que ha permitido desplegar un mercado de servicios de computación por demanda, ha adquirido gran popularidad, lo que ha llevado a que muchos directivos de empresas y departamentos de tecnología deseen adquirir soluciones basadas en ella, a pesar de que aún no tienen claro cómo deberían contratarlas. La metodología y las recomendaciones detalladas en este documento se basan principalmente en las mejores prácticas de ITIL v3, TMF (eTOM) y de definición de Acuerdos de Nivel de Servicio (ANS), teniendo en cuenta criterios como continuidad, disponibilidad, calidad y seguridad del servicio, entre otros. Los resultados obtenidos al usar el CTP (Costo Total de Propiedad) como herramienta financiera para la toma de decisiones en proyectos de tecnología dio como resultado un claro beneficio económico al optar por la computación en la nube.

Palabras clave: Computación en la Nube, Acuerdos de Nivel de Servicio, ITIL v3, eTOM.

\section{Abstract}

The Cloud Computing system is the new technology that has allowed a market deployment, able to provide any computing services on demand. Due that the technology innovation has reached a great popularity, which has led many business leaders and technology departments wishing to acquire solutions on the cloud computing, although they are not yet clear, how they should hire them. The methodology and recommendations given in this paper, are mainly based on the ITIL v3, TMF (eTOM)'s best practices, and the Service Level Agreements (SLAs)' definition, based on the Continuity, Availability, Service Quality and Security's criteria, among others.

* Universidad Nacional de Colombia (Bogotá-Cundinamarca, Colombia). baleon16@gmail.com

** MSc. Universidad Nacional de Colombia (Bogotá-Cundinamarca, Colombia). roseromario@gmail.com 
The results obtained, by using the TCO (Total Cost of Ownership) as a financial tool for making decisions in the technology projects, resulted in a clear economic benefit by opting for the cloud computing service.

Keywords: Cloud Computing, Service Level's Agreement, ITIL v3, Continuity, Availability, Quality and Security's Service, eTOM.

\section{Resumo}

AComputação em Nuvem, nova tecnologia que tornou possível a oferta de um mercado de serviços de computação por demanda, tem adquirido grande popularidade, o que tem levado a que muitos diretores de empresas e departamentos de tecnologia desejem adquirir soluções baseadas nela, apesar de que ainda não têm claro como deveriam contratá-las. A metodologia e as recomendações detalhadas neste documento se baseiam principalmente nas melhores práticas de ITIL v3, TMF (eTOM) e de definição de Acordos de Nível de Serviço (ANS), tendo em conta critérios como continuidade, disponibilidade, qualidade e segurança do serviço, entre outros. Os resultados obtidos ao usar o CTP (Custo Total de Propriedade) como ferramenta financeira para a tomada de decisões em projetos de tecnologia deu como resultado um claro benefício econômico ao optar pela computação em nuvem.

Palavras chave: Computação em Nuvem, Acordos de Nível de Serviço, ITIL v3, eTOM. 


\section{INTRODUCCIÓN}

En la actualidad, las TIC (Tecnología de Información y las Comunicaciones) juegan un papel protagónico en el día tras día del desarrollo de la sociedad, hasta el punto de convertirse en el área contemporánea de mayor dinamismo económico, técnico y jurídico; debido a esto la dependencia a las redes de comunicaciones es cada vez más fuerte, creando la necesidad de acceder a la información desde cualquier lugar, desde cualquier dispositivo, a cualquier momento y de manera segura. Las soluciones a esta necesidad se están soportando bajo el modelo de servicio en la nube (Cloud Services), el cual ha llevado a una revolución respecto a cómo los proveedores de infraestructura tecnológica deben responder a estos requerimientos de la sociedad, para hacer más fácil y provechosa la interacción con el resto del mundo.

Cloud Computing es un nuevo modelo de prestación de servicios de negocio y tecnología que incluso permite al usuario acceder a un catálogo de servicios estandarizados y responder con ellos a las necesidades de su negocio de forma flexible y adaptativa, en caso de demandas de carga de trabajo computacional no previsibles o de picos de trabajo, pagando únicamente por el consumo efectuado, o incluso gratuitamente, en caso de proveedores que se financian mediante publicidad o de organizaciones sin ánimo de lucro.

Este trabajo se realizó con la motivación de lograr un avance para la estandarización acerca de los lineamientos que una empresa, principalmente estatal, debe seguir para hacer la contratación de servicios de Computación en la Nube. Hasta el momento, el Gobierno colombiano no ha publicado un estándar, una guía o un lineamiento político, como una ley, que específicamente les indique a las empresas estatales cómo deben contratar los servicios de computación en la nube, por lo cual, a pesar de que actualmente existen ejecutivos que buscan migrar su plataforma a un modelo de servicio como el que ofrece la computación en la nube, el proceso ha sido demorado; el escaso conocimiento que se tiene acerca de cómo se debe contratar y qué lineamientos se deben seguir para que este tipo de proyectos de migración e implementación de los servicios en la nube sean exitosos es evidente.

\section{Cloud Computing}

El presupuesto cada vez más limitado de las empresas ha generado que las inversiones en infraestructura de TI se hagan de la manera más saludable para las finanzas de las empresas, de tal forma que estos gastos han ido moviéndose a servicios de outsourcing, arrendamientos o Cloud Computing. Uno de los modelos de negocio con mayor impacto en la actualidad es el Cloud Computing, que es una nueva tendencia del siguiente nivel de evolución de la Internet y la computación distribuida.

La computación en la nube se está convirtiendo en un serio cambio de paradigma de la forma como usamos los ordenadores; se basa en varias tecnologías, que no son nuevas, sin embargo, la creciente disponibilidad de ancho de banda permite nuevas combinaciones y abre nuevas perspectivas de TI. La computación en la nube es una tendencia emergente que ofrece recursos de computación prácticamente ilimitados y bajo demanda.

Debido a la novedad de este tipo de tecnología, actualmente existe gran desconocimiento en las empresas respecto a cómo pueden adquirir y contratar este tipo de servicios para ponerlos a su disposición; por ello en este trabajo se va a implementar una metodología y se formularán recomendaciones que sirvan de guía para que las empresas conozcan más en detalle cómo el Cloud Computing y los Proveedores de Servicio pueden cambiar su forma de satisfacer los requerimientos de Infraestructura de Tecnología (IT), incluyendo pautas para identificar cómo definir los servicios por contratar y sus niveles de servicio; además, se indicará cómo hacer un análisis para obtener la justificación económica, usando el Costo Total de Propiedad (TCO, por sus siglas en inglés) como una herramienta para evaluar la viabilidad de los proyectos de TI.

Las principales fuerzas impulsoras detrás de la aparición de Cloud Computing son el exceso de demanda de capacidad de los centros de datos de las grandes empresas de hoy. "Las organizaciones buscan en Cloud Computing una utilidad para reducir los costos y aumentar la eficacia de las TIC, y se tratan de pasar de las inversiones fijas de gastos de capital 
a gastos de operación variables, y así soportar los procesos informáticos de las empresas" [1].

A continuación se describen las tres capas de la pirámide (Figura 1):

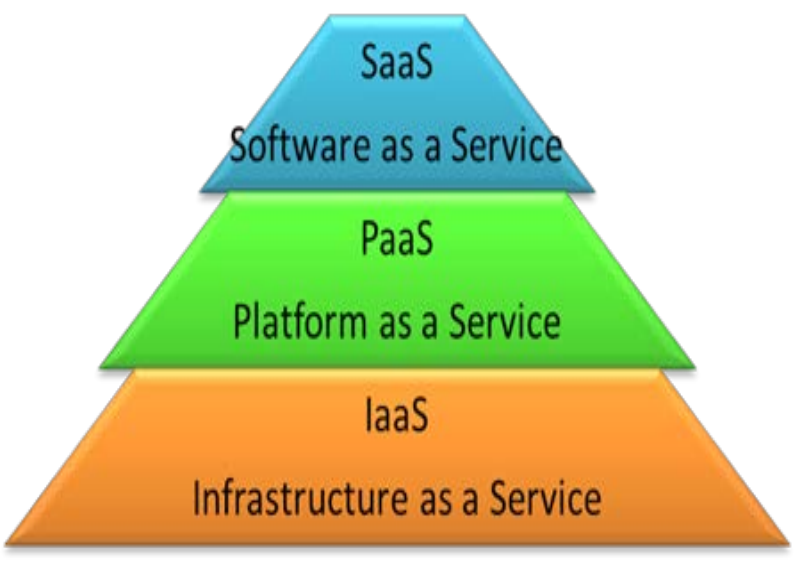

Fig. 1. Capas de los servicios ofrecidos en Cloud Computing

"El software como servicio (en inglés software as a service, SaaS) se encuentra en la capa más alta y caracteriza una aplicación completa ofrecida como un servicio, por-demanda, vía multitenencia —que significa una sola instancia del software que corre en la infraestructura del proveedor y sirve a múltiples organizaciones de clientes-" [1]. "El ejemplo de SaaS más conocido es Salesforce.com, se incluyen las Google Apps, que brindan servicios primordiales de negocio como el correo (e-mail). La aplicación multitenencia de Salesforce.com es el mejor ejemplo de una solución en la nube. Otro ejemplo es la plataforma MS Office como servicio SaaS, con su denominación de Microsoft Office 365, que incluye versiones online de la mayoría de las aplicaciones de esta suite ofimática de Microsoft" [1]. A pesar de ser la solución más completa, se debe precisar que la misma aplicación se usa para varios usuarios simultáneamente; cada usuario tiene limitaciones para configurar o personalizar la aplicación.

La segunda capa, la "plataforma como servicio (en inglés Platform as a Service, PaaS), es la encapsulación de una abstracción de un ambiente de desarrollo y el empaquetamiento de una serie de módulos o complementos que proporcionan, normalmente, una funcionalidad horizontal (persistencia de datos, autenticación, mensajería, etc.). De esta forma, un arquetipo de plataforma como servicio podría consistir en un entorno conteniendo una pila básica de sistemas, componentes o APIs preconfiguradas y listas para integrarse sobre una tecnología concreta de desarrollo [...]. Las ofertas de PaaS pueden dar servicio a todas las fases del ciclo de desarrollo y pruebas del software, o pueden estar especializadas en cualquier área en particular, tal como la administración del contenido" [1].

"La infraestructura como servicio (Infrastructure as a Service, IaaS) -también llamado en algunos casos hardware as a service, HaaS)- se encuentra en la capa inferior y es un medio de entregar almacenamiento básico y capacidades de cómputo como servicios estandarizados en la red. Servidores, sistemas de almacenamiento, conexiones, enrutadores y otros sistemas se concentran (por ejemplo a través de la tecnología de virtualización) para manejar tipos específicos de cargas de trabajo — desde procesamiento en lotes ("batch") hasta aumento de servidor/almacenamiento durante las cargas pico-_" [1]. La oferta comercial más conocida es Amazon Web Services, por sus servicios EC2 para cómputo, y S3 para almacenamiento.

\section{ITIL V3}

"ITIL (Biblioteca de infraestructura de Tl) es una serie de publicaciones exhaustivas y consistentes que se utilizan para describir y optimizar un marco de trabajo para la gestión de calidad de servicio $\mathrm{Tl}$ dentro de una organización, alineado con el estándar internacional, ISO/IEC 20000. La filosofía de ITIL es globalmente reconocida como la fundación de las mejores prácticas de la Gestión de Servicio Tl, respaldado por un programa de calificación profesional” [2].

La última versión de ITIL (v3) [2] consiste en un núcleo de cinco publicaciones que reemplaza la versión previa de ITIL (publicada en el 2000). Las publicaciones núcleo, que proveen la guía necesaria para un acercamiento integrado como lo requiere el estándar internacional ISO/IEC20000, son:

- Estrategia de servicio

- Diseño de servicio

- Transición de servicio 
- Operación de servicio

- Mejoramiento continuo de servicio

Los volúmenes centrales vienen acompañados de títulos complementarios, personalizados para mercados particulares o contextos tecnológicos.

Es importante destacar que los servicios de $\mathrm{Tl}$ se están alineando e integrando a los negocios, y las mejores prácticas de ITIL v3 buscan un acercamiento de gestión de negocios y de un método para la Gestión de Servicio $\mathrm{Tl}$, destacando los aspectos adicionales para ejecutar el departamento de $\mathrm{Tl}$ como un negocio.

"La Gestión de Servicio es un conjunto de habilidades organizacionales especializadas en proveer valor para los clientes en forma de servicios. El núcleo de la Gestión de Servicio es transformar los recursos en servicios de valor" [2].

\section{GESTIÓN Y ACUERdos DE NIVEL DE SERVICIO}

Continuando con la aplicación de las mejores prácticas, la entidad que vaya a contratar Servicios de Computación en la Nube debe definir muy bien cuáles son los Acuerdos de Nivel de Servicio (ANS) que mejor se ajustan a sus necesidades. Por lo cual, se van a detallar cuáles son las exigencias que se deben hacer para los Acuerdos y Gestión de Nivel de Servicio (Service Level Agreements -SLA- and Management).

La manera como las empresas negocian, crean y gestionan sus acuerdos de nivel de servicio (SLA) está experimentando cambios rápidos hoy en día. Cada vez más gerentes reconocen la importancia de los acuerdos de nivel de servicio en la definición de los niveles aceptables de servicio de TI, y las organizaciones líderes están yendo mucho más allá, no contentas con medir el desempeño de TI de manera simplista, señalando factores tales como si un sitio está arriba o abajo, vigilan los procesos claves del negocio en toda la empresa, los cuales tienen un impacto real en los ingresos y beneficios. "Los líderes ven los ANS y la gestión a nivel de servicio como un paso crítico que conduce hacia una verdadera alineación entre los grupos de TI, proveedores de servicios externos y la gestión empresarial" [3].

\section{TeCnOlogía EMPLEada PaRa las SOLUCIONES DE COMPUTACIÓN EN LA NUBE}

En esta sección del artículo se van a dar una serie de detalles específicos acerca de cómo debe aplicarse este conocimiento a soluciones reales que se encuentran en el mercado actual; esto permitirá a los clientes hacer solicitudes con mayor nivel de detalle, lo cual les permitirá seleccionar el proveedor más apropiado a sus necesidades.

Los datacenter están clasificados en TIERs o niveles, según el estándar del Uptime Institute, que guía el diseño y las inversiones en data centers y es utilizada para determinar la disponibilidad de una instalación; éstos son:

- Tier $\mathbf{1}=$ Componentes no redundantes (enlaces y servidores). Garantiza disponibilidad de $99,671 \%$.

- Tier 2=Tier $1+$ Componentes redundantes. Garantiza disponibilidad de 99,741\%.

- Tier 3=Tier $2+$ Equipos con alimentación energética dual y varios enlaces. Garantiza disponibilidad de $99,982 \%$.

- Tier 4=Tier $3+$ Tolerancia a fallas de todos los componentes, incluyendo enlaces, equipos de almacenamiento, refrigeración, sistemas de climatización, servidores, etc. Todos los equipos son de alimentación energética dual. Garantiza disponibilidad de $99,995 \%$.

La entidad de gobierno debe solicitar a su proveedor de servicio el TIER en cual está clasificado el datacenter que va a contener la operación del servicio que se está contratando; para esto se recomienda que al menos el TIER del proveedor de servicio sea de Nivel 3. Un datacenter TIER 3 ó 4 garantiza la correcta operación del servicio que se está contratando en la nube. Es importante destacar que la disponibilidad indicada aplica únicamente para el Hardware que hace parte de la Infraestructura de Tecnología. De acá en adelante, dependiendo del tipo de servicio que se desee contratar (IaaS, PaaS o SaaS), el ANS que hace referencia a la disponibilidad del servicio irá añadiendo componentes a la cadena que soporta el servicio, por lo cual la disponibilidad irá bajando su calidad, al combinar 
las disponibilidades ofertadas por cada componente incluido en la solución, como son: Hardware, Redes de comunicaciones, interconexiones, Sistema Operativo, Aplicaciones, hasta llegar a la experiencia de usuario, que hace uso del servicio desde una interfaz a través un portal web.

En el caso de que el servicio contratado sea SaaS (Software as a Service), se recomienda que se exija al proveedor de servicios una disponibilidad para Producción de $99.5 \%$ en una base mensual, es decir, el servicio no debe estar abajo más de 3,6 horas en una base mensual. También se podrá aceptar un nivel de servicio que ofrezca una disponibilidad del SaaS de $99 \%$, es decir, no se aceptará que el servicio ofrecido tenga tiempos de caída superiores a 7,2 horas en una base mensual.

En caso de que el nivel de servicio ofrecido por el proveedor de servicios SaaS tenga una disponibilidad inferior al $99 \%$ en una base mensual, la entidad que contrate el servicio no lo aceptará como un servicio de calidad, por lo cual deberá exigir al proveedor de servicio el cumplimiento de los ANS estipulados en el contrato, tanto con el objetivo de exigir una mejora en el servicio como también de hacer el cobro de los descuentos que se estipulen en el contrato.

El proveedor de servicio deberá mostrar evidencia a la entidad de que está buscando la causa raíz del problema y de que se está atendiendo con el objetivo de corregir el problema. Las multas aplicadas, por ejemplo, podrán ser un número definido de días de crédito de tarifas por el SaaS aplicable con base en las tarifas mensuales incurridas. Estos créditos serán aplicados al cliente en el próximo período facturable que se realice por el Proveedor de Servicio. En caso de que las fallas no sean resueltas y se sigan presentando de manera consecutiva dentro de un período de tres meses, el cliente deberá entrar a analizar si se continúa contratando el servicio con el proveedor actual, o si, por el contrario, da por terminado el contrato.

En el caso de la Infraestructura como Servicio (IaaS) la cobertura de los ANS se puede estipular por cada una de las capas de hardware. Al estar administrando una infraestructura de TI, con los ANS se deben tener en cuenta las siguientes especificaciones para los servicios: Grado de cumplimiento, calidad y rigurosidad de la solución del proponente según los alcances, características, ventana de servicio, horario de soporte y niveles de servicio para cada servicio.

\section{- Descuentos}

Por incumplimiento de los servicios se deben aplicar descuentos a las facturas mensuales del Proveedor de Servicios, según el grado de falla en los niveles de servicio. A continuación se detalla una estrategia recomendada para el cálculo de descuentos que aplica para el incumplimiento de los Acuerdos de Nivel de Servicio:

- Aplicación del grado de cumplimiento de los niveles de servicio individuales con un peso a cada uno de los servicios a fin de determinar si aplica el descuento, donde la suma de pesos de los servicios da un total de 100 puntos.

- Se utiliza un indicador y su nivel de servicio para determinar el grado de cumplimiento de cada servicio, según lo muestra la Tabla 1: 


\section{TABLA 1}

TABLA DE EVALUACIÓN DE GRADO DE CUMPLIMIENTO POR SERVICIO.

\begin{tabular}{|c|c|c|c|}
\hline Servicio & $\begin{array}{l}\text { Nivel de servicio } \\
\text { (NS) requerido }\end{array}$ & $\begin{array}{l}\text { Peso* } \\
\text { (P) }\end{array}$ & $\begin{array}{l}\text { Grado de cumplimiento (GC) por } \\
\text { servicio según el NS resultante** }\end{array}$ \\
\hline \multirow[t]{3}{*}{ 1.Telecomunicaciones } & \multirow{3}{*}{$\begin{array}{l}\text { Disponibilidad } \\
\text { de enlaces } \geq 99.5 \%\end{array}$} & \multirow{3}{*}{15} & $99.5 \% \leq \mathrm{NS} \leq 100 \% ; \mathrm{GC}=100 \%$ \\
\hline & & & $98.0 \% \leq \mathrm{NS} \leq 99.49 \% ; \mathrm{GC}=80 \%$ \\
\hline & & & $96.5 \% \leq \mathrm{NS} \leq 97.99 \% ; \mathrm{GC}=60 \%$ \\
\hline \multirow{3}{*}{ 2. Red de datos } & \multirow{3}{*}{ Disponibilidad $\geq \% ৭ 9,0$} & \multirow{3}{*}{10} & $99.5 \% \leq \mathrm{NS} \leq 100 \% ; \mathrm{GC}=100 \%$ \\
\hline & & & $98.0 \% \leq \mathrm{NS} \leq 99.49 \% ; \mathrm{GC}=80 \%$ \\
\hline & & & $96.5 \% \leq \mathrm{NS} \leq 97.99 \% ; \mathrm{GC}=60 \%$ \\
\hline \multirow{3}{*}{ 3. Red de telefonía } & \multirow{3}{*}{ Disponibilidad $\geq \% ৭ \wedge$} & \multirow{3}{*}{12} & $98.0 \% \leq \mathrm{NS} \leq 100 \% ; \mathrm{GC}=100 \%$ \\
\hline & & & $96.5 \% \leq \mathrm{NS} \leq 97.99 \% ; \mathrm{GC}=80 \%$ \\
\hline & & & $95.0 \% \leq \mathrm{NS} \leq 96.49 \% ; \mathrm{GC}=60 \%$ \\
\hline \multirow{3}{*}{ 4. Red de voz sobre IP } & \multirow{3}{*}{ Disponibilidad $\geq \% ৭ 9,0$} & \multirow{3}{*}{12} & $99.5 \% \leq \mathrm{NS} \leq 100 \% ; \mathrm{GC}=100 \%$ \\
\hline & & & $98.0 \% \leq \mathrm{NS} \leq 99.49 \% ; \mathrm{GC}=80 \%$ \\
\hline & & & $96.5 \% \leq \mathrm{NS} \leq 97.99 \% ; \mathrm{GC}=60 \%$ \\
\hline \multirow{3}{*}{ 5. Internet } & \multirow{3}{*}{ Disponibilidad $\geq \% ৭ ৭, १$} & \multirow{3}{*}{15} & $99.9 \% \leq \mathrm{NS} \leq 100 \% ; \mathrm{GC}=100 \%$ \\
\hline & & & $98.5 \% \leq \mathrm{NS} \leq 99.98 \% ; \mathrm{GC}=80 \%$ \\
\hline & & & $97.0 \% \leq \mathrm{NS} \leq 98.49 \% ; \mathrm{GC}=60 \%$ \\
\hline \multirow{3}{*}{ 6. Plataforma colaborativa } & \multirow{3}{*}{ Disponibilidad $\geq \% ৭ 9,9$} & \multirow{3}{*}{10} & $99.9 \% \leq \mathrm{NS} \leq 100 \% ; \mathrm{GC}=100 \%$ \\
\hline & & & $98.5 \% \leq \mathrm{NS} \leq 99.98 \% ; \mathrm{GC}=80 \%$ \\
\hline & & & $97.0 \% \leq \mathrm{NS} \leq 98.49 \% ; \mathrm{GC}=60 \%$ \\
\hline \multirow{3}{*}{ 7. Acceso a aplicaciones } & \multirow{3}{*}{ Disponibilidad $\geq \% ৭ ৭, 9$} & \multirow{3}{*}{12} & $99.9 \% \leq \mathrm{NS} \leq 100 \% ; \mathrm{GC}=100 \%$ \\
\hline & & & $98.5 \% \leq \mathrm{NS} \leq 99.98 \% ; \mathrm{GC}=80 \%$ \\
\hline & & & $97.0 \% \leq \mathrm{NS} \leq 98.49 \% ; \mathrm{GC}=60 \%$ \\
\hline \multirow{4}{*}{ 8. Web Server } & \multirow{3}{*}{ Disponibilidad $\geq \% ৭ 9,9$} & \multirow{3}{*}{14} & $99.9 \% \leq \mathrm{NS} \leq 100 \% ; \mathrm{GC}=100 \%$ \\
\hline & & & $98.5 \% \leq \mathrm{NS} \leq 99.98 \% ; \mathrm{GC}=80 \%$ \\
\hline & & & $97.0 \% \leq \mathrm{NS} \leq 98.49 \% ; \mathrm{GC}=60 \%$ \\
\hline & & 100 & \\
\hline
\end{tabular}

*Los valores usados en la tabla son a modo de ejemplo; la cantidad de servicios, peso, Nivel de Servicio y grado de cumplimiento serán determinados por el cliente en conjunto con el proveedor de servicio.

- Para determinar el nivel global de cumplimiento se utiliza la fórmula siguiente, que consiste en el promedio ponderado del grado de cumplimiento individual de cada uno de los servicios:

GC Global $=$ GC1 $\times$ P1 + GC2 $\times$ P2 $+\ldots+$ GCn $\times$ Pn (donde $\mathrm{n}$ es el número de servicios)
En tal sentido, el descuento en la facturación de los servicios se aplica de acuerdo con la gravedad de la falta, determinado por el Grado de Cumplimiento Global: 


\section{TABLA 2}

Descuentos APLICADOS SEGÚN GRADO DE CUMPLIMIENTO

\begin{tabular}{|c|c|}
\hline Grado de cumplimiento global & $\begin{array}{c}\text { Descuento en } \\
\text { factura }\end{array}$ \\
\hline entre 96 y 100 & $0 \%$ \\
\hline entre 90 y 95 & $2 \%$ \\
\hline entre 85 y 89 & $5 \%$ \\
\hline menos de 85 & $7 \%$ \\
\hline
\end{tabular}

* Los valores usados en la tabla son a modo de recomendación; el grado de cumplimiento global y el descuento en la factura se negociarán entre el cliente y el proveedor de servicios.

- El descuento se aplicará mensualmente, descontando el porcentaje indicado en la próxima factura global de servicios.
El proveedor de servicios confirmará que acepta la metodología para la aplicación de los descuentos. Si el proveedor no acepta, indicará el motivo y ofrecerá una alternativa al cliente. El descuento formará parte integral del contrato de servicio entre el Proveedor de Servicio y el cliente y no podrá ser ajustado luego de la firma del contrato.

Si el Proveedor de Servicio desea proponer alguna otra alternativa que logra este mismo objetivo en el cliente, el enfoque será explicado y debe proporcionar su impacto en el negocio del cliente, de tal manera que al final cliente y proveedor lleguen a un acuerdo.

De igual manera, se recomienda que si ocurren tres incumplimientos por debajo de $85 \%$ durante 12 meses consecutivos sean revisados los términos del contrato, lo cual podrá resultar en su terminación.

\section{- Matriz de probabilidad vs. impacto}

\section{TABLA 3}

MATRIZ DE PROBABILIDAD VS. IMPACTO [4]

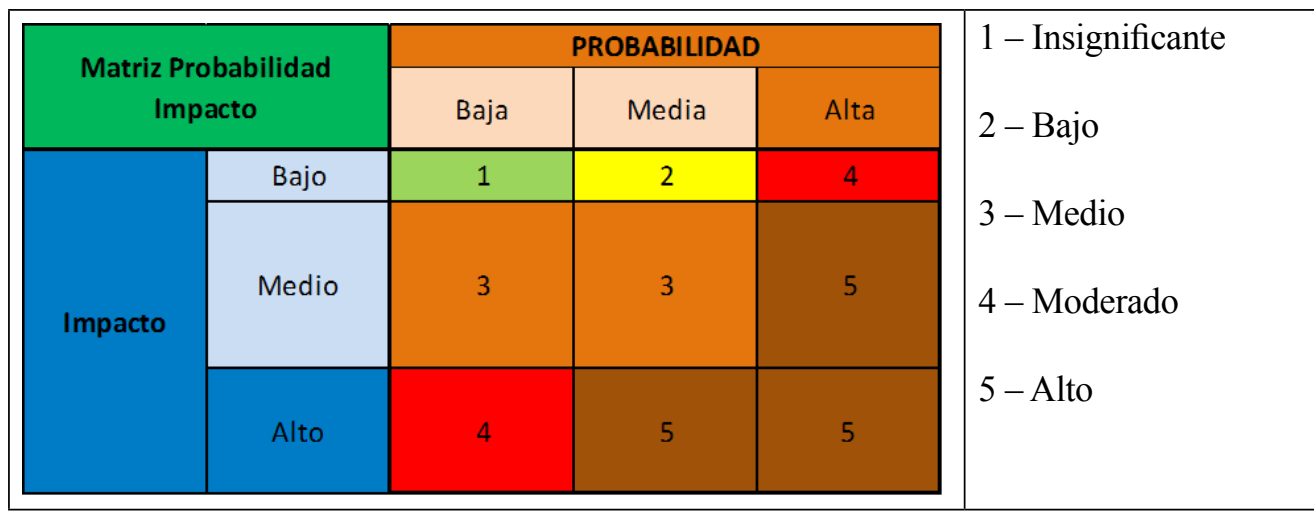

"Esta matriz es una herramienta para analizar los eventos futuros, previamente identificados, utilizando las dos principales dimensiones del riesgo: Probabilidad vs. Impacto. Basado en las combinaciones de escalas de la probabilidad y del impacto se construye una matriz para asignar calificaciones al riesgo" [4], como se observa en la Tabla 3.

Una vez que se haya realizado el proceso de evaluación, las amenazas y las oportunidades se ordenan según la calificación de su importancia y, por consiguiente, por su prioridad. El ordenamiento de prioridades solo es aproximado, debido a que los números utilizados para crear la lista son estimaciones (depende de la calidad de los datos).

El cliente es quien decidirá hacer o no este análisis, ya que su aplicación depende del tiempo y el presupuesto disponible, así como de la necesidad del planteamiento cualitativo o cuantitativo acerca de los riesgos y los impactos. El proceso de análisis cuantitativo de riesgos ayuda a analizar numéricamente la probabilidad de los riesgos priorizados y sus consecuencias. 


\section{- Análisis del Valor Monetario Esperado (EVM)}

"EVM es un concepto estadístico que calcula el resultado promedio cuando el futuro incluye escenarios que pueden ocurrir o no (análisis con incertidumbre). Es la sumatoria del producto del valor de cada posible resultado (impacto o la cantidad en juego) por su probabilidad de ocurrencia. El impacto de las oportunidades generalmente se expresará con valores positivos, mientras que el de los riesgos será negativo" [5].

Los riesgos deben ser identificados por el cliente y el proveedor de servicios, quienes deben asignar a cada servicio una probabilidad de ocurrir de la manera más fiable posible. Los riesgos que podrán identificarse son: Incendio en el datacenter principal, caída del servidor de aplicaciones core, caída de los servicios de telecomunicaciones, robo de la información, ataque de un virus crítico que dañe el sistema, terremoto o desastre natural que afecte la operación, corte en el servicio de energía, falla de Hardware que afecte servidores y almacenamiento, entre otros.

A estos riesgos se les debe asignar un valor de probabilidad de que suceda, y luego se procede a hacer la operación correspondiente para encontrar el valor de EVM para el escenario seleccionado; el objetivo de usar el EVM es que se logre seleccionar el escenario más apropiado (el que tenga menor EVM); este valor EVM se toma como un punto de referencia para asegurar que los costos de mitigar estos riesgos no sean mayores que el valor de asumir los consecuencias de que ocurran sin tener ninguna contingencia.

\section{Costo total DE PROPIEDAD}

"El Costo total de propiedad (Total Cost of Ownership o TCO) es el costo total de proveer y mantener una tecnología y les permite a los usuarios conocer los costos directos e indirectos, así como los beneficios de la compra de equipos o programas" [6]. Este análisis permitirá a los clientes identificar todos los costos incurridos en la solución de TI a lo largo de su ciclo de vida, y así decidir por la opción de menor costo y de mayor beneficio para el negocio.

La base para la evaluación es el Costo Total de Propiedad (CTP), definido como el valor presente neto de todos los costos asociados a la adquisición, implantación, estabilización, soporte, mantenimiento, capacitación y otros conceptos requeridos para garantizar la sostenibilidad del sistema en el tiempo.

El cálculo del CTP se hará con base en un período de cinco años, contado a partir del inicio del contrato, y utilizando una Tasa de Descuento (TD) equivalente a $1.8 \%$ efectivo mensual; este valor varía de acuerdo con cada compañía, ya que dependerá de la rentabilidad del dinero y de las decisiones del área de finanzas.

En donde,

$\mathbf{n}=$ número de períodos de duración del proyecto. En este caso, $\mathrm{n}$ es igual a 60 , equivalente a los meses de duración del proyecto (5 años).

$\mathbf{i}$ = número del período, que va de 0 hasta el número $\mathrm{n}$ de períodos de duración del proyecto.

$\mathbf{j}=$ tipo de costo. El número de tipos de costos dependerá de las clases de costos que relacione el oferente en su propuesta.

$\mathbf{C i j}=$ Costo de propiedad del tipo $\mathbf{j}$, pagado en el mes i.

El oferente debe incluir todos los costos asociados al soporte, mantenimiento y sostenibilidad de la infraestructura, debidamente discriminados.

A continuación se detallará un ejercicio de TCO para identificar bajo qué circunstancias una solución de Cloud Computing bajo el modelo de IaaS (Infraestructura como Servicio) puede ser la opción más económica, comparada con la forma tradicional de compra de infraestructura de tecnología. Se presenta este ejemplo como medio ilustrativo para la decisión de la compra versus el pago mensual en la nube de un servidor de 16 Cores, 32 GB de RAM y 3200 GB de espacio en disco. 


\section{TABLA 4}

\section{COSTOS ASOCIADOS A LA COMPRA TRADICIONAL DE IT}

\begin{tabular}{|l|c|}
\hline \multicolumn{1}{|c|}{ Costo } & \$ Valor (US) \\
\hline $\begin{array}{l}\text { El costo de instalación, configuración, mantenimiento, actualización } \\
\text { de software, etc. }\end{array}$ & US\$ 1.000 pago en el período N. ${ }^{\circ} \cdot$ \\
\hline $\begin{array}{l}\text { El costo del hardware, incluyendo como mínimo los sistemas de } \\
\text { respaldo, servidores y el reemplazo por vejez o daño, etc. }\end{array}$ & US\$ 5.500 pago en el período No 0. \\
\hline $\begin{array}{l}\text { El costo del espacio físico de un centro de cómputo, racks, servidores, } \\
\text { aire, UPS, centro de datos alterno, etc. }\end{array}$ & $\begin{array}{c}\text { US\$ 25 costo fijo mensual por cada mes } \\
\text { de duración del proyecto }\end{array}$ \\
\hline $\begin{array}{l}\text { El costo del personal requerido para el mantenimiento del software } \\
\text { del sistema y el costo de entrenamiento de nuevo personal funcional } \\
\text { y técnico, etc. }\end{array}$ & $\begin{array}{c}\text { US\$ 75 costo fijo mensual por cada mes } \\
\text { de duración del proyecto }\end{array}$ \\
\hline $\begin{array}{l}\text { El costo de las actualizaciones, configuraciones del sistema, la base } \\
\text { de datos, copias de respaldo y restauración, etc. }\end{array}$ & $\begin{array}{c}\text { US\$ } 500 \text { costo generado una vez y al } \\
\text { final de cada año }\end{array}$ \\
\hline Otros: Extensión de garantía del HW. & $\begin{array}{c}\text { US\$ 2.500 costo a pagar al cumplir 36 } \\
\text { meses de proyecto }\end{array}$ \\
\hline \multicolumn{1}{|c|}{ Costo Total de Propiedad } & US\$ 12.424 \\
\hline
\end{tabular}

Bajo el modelo tradicional de compra de Infraestructura Tecnológica el cliente deberá pagar el total de los US\$ 6.500 en el período de inicio del proyecto, y durante cinco años (60 meses) deberá pagar un costo total de propiedad de US\$12.424.

Si en lugar de hacer la compra tradicional de equipos ejecutamos el proyecto a través de un proveedor de servicios en la nube, el costo mensual de estos recursos es de US\$278; este valor contempla los valores de operación y mantenimiento para los recursos contratados durante un período de proyecto de 5 años, con lo cual se observa que el proveedor del servicio de IaaS genera un margen de ganancia para su negocio. El flujo de pagos para cada uno de los proyectos se muestra en la Figura 2.

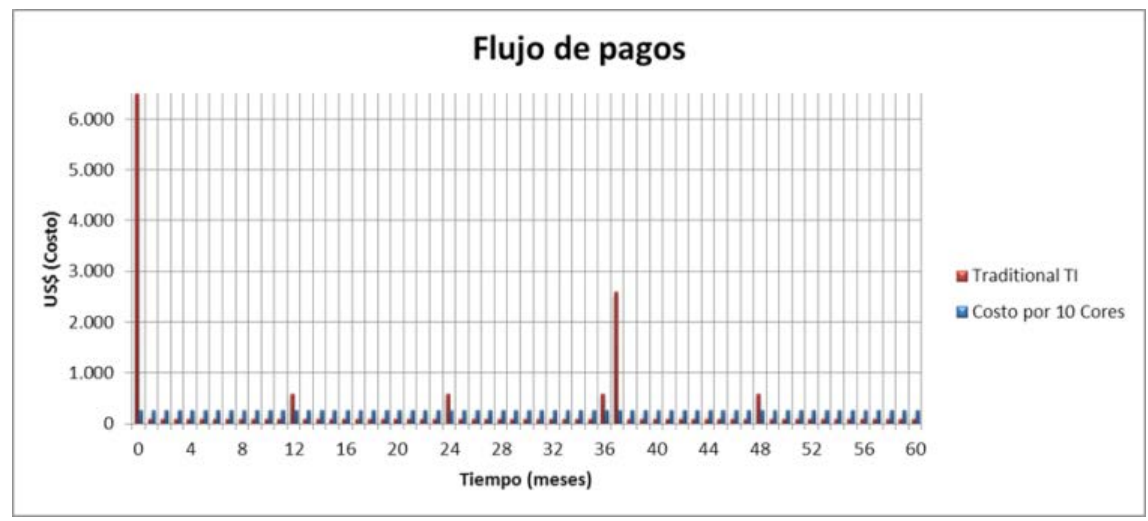

Fig. 2. Flujo de Pagos en proyecto de TI

Se hizo el cálculo del TCO para cada uno de los proyectos, y dio como resultado que el mejor TCO es para la opción de compra de IT a través de un proveedor de servicios en la nube, ya que su TCO es
US\$10.243, por lo cual hay una reducción del TCO en $18 \%$ respecto a la compra tradicional; este valor puede variar según la tasa de descuento aplicada. 
Debido a que la Tasa de descuento (TD) es determinada por el cliente, en la tabla a continuación se va a mostrar el TCO hecho con la tasa de 1.8\% E.M y otro ejercicio con $0.9 \%$ E.M, en el cual se muestra cual es el ahorro o mejora en el TCO al adquirir el servicio en la nube en lugar de hacer la compra tradicional de la infraestructura de tecnología.

\section{TABLA 5}

AnÁlisis TCO -Servicio en la Nube Vs. COMPRA tradicional de IT-

\begin{tabular}{|c|c|c|c|}
\hline \multicolumn{4}{|c|}{ Duración del proyecto 60 meses } \\
\hline TD & TCO & TCO & Ahorro \\
(Tasa de & Servicio en la & Compra Tradicional de & \\
Descuento) & Nube & IT & $\%$ \\
\hline $1.8 \%$ E.M & $\$ 10,243$ & $\$ 12,424$ & $17.6 \%$ \\
\hline $0.9 \%$ E.M & $\$ 13,006$ & $\$ 14,326$ & $9.2 \%$ \\
\hline
\end{tabular}

En la Figura 3 vamos a mostrar cómo el TCO se comporta en la medida que va cambiando la cantidad de recursos solicitados al proveedor de servicios. El valor de cada TCO detallado en la figura se hizo teniendo en cuenta un período de duración del proyecto de 5 años. En la Figura 3 se observa cómo el TCO para la compra tradicional de TI es igual, sin importar la cantidad de recursos consumidos, ya que sean usados o no la máquina es propiedad del cliente y deberá asumir el costo total de propiedad y asumir altos costos por subutilización de los recursos.

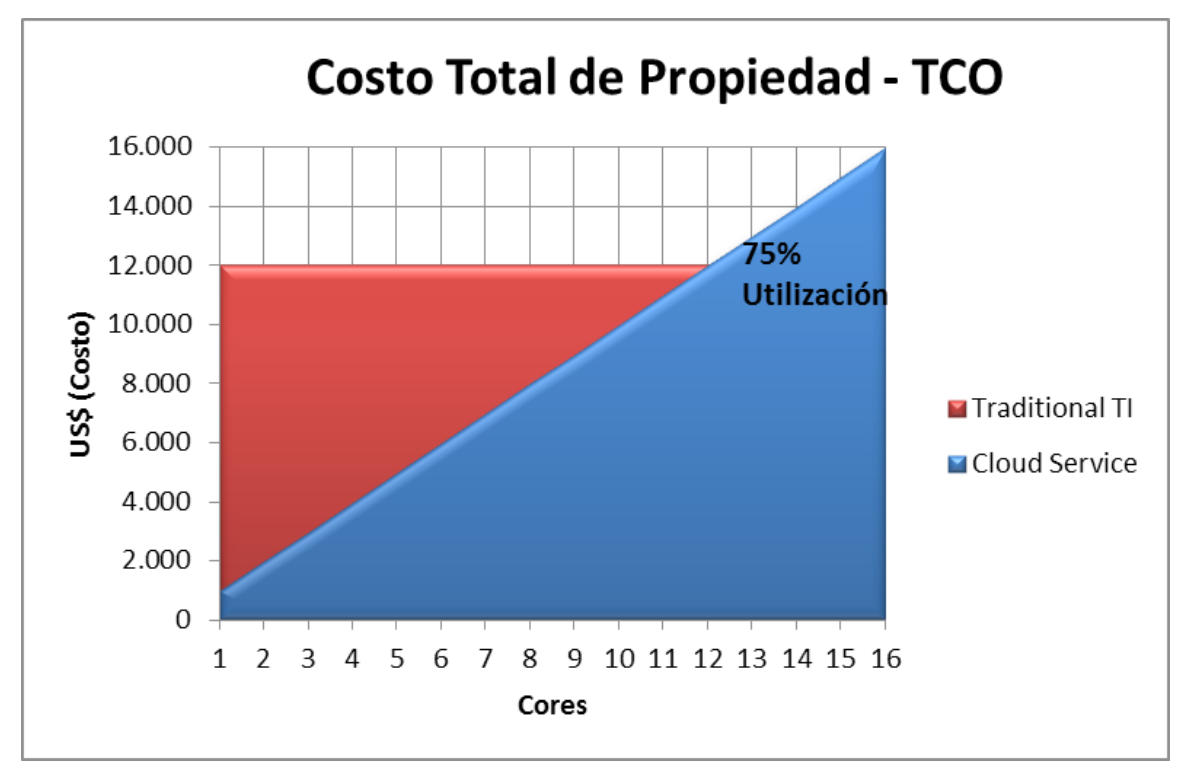

Fig. 3. TCO - Cloud Computing vs. Tradicional TI

Al contratar los servicios de IaaS con un proveedor en la nube, el cliente tiene la opción de pagar únicamente por los recursos que usa para operar y mantener su negocio con la opción de ir creciendo según los requerimientos de TI. Para este caso particular vemos que el TCO para ambas maneras de ejecución del proyecto llega a un punto de equilibrio cuando la utilización del servidor es de $75 \%$ durante todo el tiempo de duración del proyecto. Este valor de $75 \%$ es realmente alto, ya que es muy difícil que un cliente 
mantenga un promedio de operación de la máquina a ese nivel; por lo cual vemos que si trabajamos con la opción de adquirir el Servicio en la nube en la modalidad de IaaS le permite a la empresa ahorrar costos debido a la subutilización de recursos de tecnología, ya que de esta manera la empresa solo deberá asumir los costos de la infraestructura que usa y podrá ir modificando la cantidad de recursos solicitados con base en la demanda del negocio.

Ahora se analizará la acumulación de pagos en función del tiempo, tomando como base una utilización de 10 Cores. Al hacer la compra de la infraestructura de manera tradicional, el cliente debe asumir un costo de adquisición (TCA -Total Cost of Acquisition-) del servidor de US\$6.500, un costo de mantenimiento y operación de US $\$ 100$ mensuales, un costo anual adicional de US\$500 por renovación de licencias y soporte de Software, y al final del año 3 deberá asumir un costo adicional de US $\$ 2.500$ para extender la garantía del hardware por 2 años adicionales, para así extender la duración del proyecto a 5 años. Este flujo de pagos se puede detallar en la Figura 4.

En el caso de adquirir los recursos de TI a través de un proveedor de Servicios en la nube, el cliente deberá únicamente asumir un costo mensual de US\$278 por la utilización de los recursos contratados; así, el cliente no tendrá que hacerse responsable del soporte ni del mantenimiento del hardware, y tampoco deberá renovar licencias ni soporte del software de la máquina; esto le permitirá al cliente optimizar sus procesos, ya que el proveedor de servicios será el encargado de entenderse con el fabricante del hardware y del software para la administración del contrato, y no tendrá que hacerlo el cliente de la manera como tradicionalmente lo venía haciendo, lo cual hará que se enfoque en el Core u objetivos principales de su negocio, sacando así mayor provecho de su recurso humano y de capital.

De acuerdo con la Figura 3, ejecutar el proyecto como servicio no requiere realizar una inversión inicial de capital alto CAPEX (Capital Expenditure), pues al adquirir la plataforma en modalidad IaaS todos los gastos se pueden ver como costos operativos OPEX (Operational Expenditure), lo cual genera mayor bienestar financiero para la compañía al evitar grandes valores de inversión para la adquisición de tecnología. La compañía del cliente también se ve beneficiada en el sentido de que disminuye sus riesgos de operación al cederlos a un tercero, y además elimina el cargo financiero de tener que asumir las pérdidas que ocasiona la obsolescencia tecnológica, ya que estos temas también comienzan a ser asumidos por el prestador del servicio.

En la Figura 4 se muestra la acumulación de los pagos realizados en el tiempo, sin tener en cuenta la tasa de descuento; de esta manera se ve la acumulación de pagos que el cliente va haciendo a lo largo del proyecto, observándose siempre que este total de pagos tiene un valor más alto en la compra tradicional de IT, comparado con el modelo de Cloud Computing.

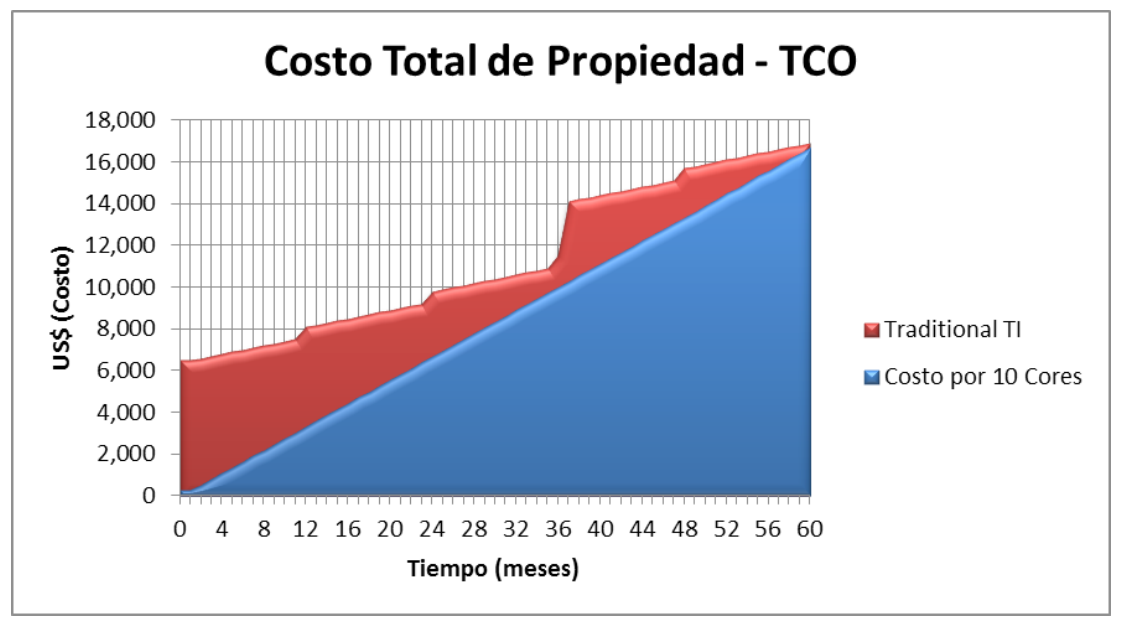

Fig. 4. TCO en el tiempo - Cloud Computing vs. Tradicional TI 
El análisis detallado en esta sección es un fuerte argumento de negocio que les puede permitir a los tomadores de decisiones orientarse hacia una solución de Cloud Computing de acuerdo con las características propias de sus infraestructuras de tecnología. Para este caso particular se usó un margen de $60 \%$ para mostrar que el negocio del proveedor de servicios es rentable $\mathrm{y}$, además, disminuye el costo total de propiedad que debe asumir un cliente, así que se puede clasificar como una relación gana-gana. En el mercado se pueden encontrar proveedores de servicios que tienen mayores márgenes de ganancia, que pueden oscilar entre un $100 \%$ y $150 \%$ y aun así ofrecen soluciones que van a traducirse en ahorro en el costo total de propiedad (TCO) para sus clientes.

A continuación se va a hacer un ejercicio de TCO usando las herramientas que actualmente ofrece de manera gratuita el Proveedor de Servicios Amazon; así como este proveedor, otros también ofrecen estas calculadoras para uso gratuito, como Softlayer, Cisco y HP, entre otros.

Los resultados que se obtuvieron usando la calculadora de TCO de Amazon son aún más inclinados hacia soluciones de Cloud Computing; se ven diferencias mucho más elevadas debido a los altos costos del alojamiento de los servidores, mientas que este valor es nulo en el ambiente de Cloud Computing. La calculadora de TCO empleada analiza las siguientes fuentes de costos: servidores, almacenamiento, redes, ambiente de datacenter y administración. En este ejemplo en particular se observa que si se compran las máquinas, la inversión en el primer año debería ser US\$84.802, pero si esa misma solución se compra como servicio a través AWS el valor de la inversión disminuye a US\$11.128, lo cual representa un ahorro para la compañía de US\$73.674, y reducción de TCO en $87 \%$. Respecto a este ejercicio en particular, se observa que el costo anual por Environment (Ambiente de Datacenter), según Amazon, le representa a un cliente US\$31.000 anuales, que es un valor elevado en relación con el costo de las máquinas, por lo cual solo se va a analizar la variación del TCO anual para el ítem Servers (servidores), donde se observa un ahorro de US\$3.459, que equivale a una reducción del TCO en $43 \%$.

Los análisis realizados hasta este punto claramente permiten a las compañías inclinarse de manera sólida hacia una solución de Cloud Computing, que es arrendamiento de tecnología como servicio y no la adquisición total de esta tecnología. Los gerentes del área de Finanzas o CFO de las compañías son cada vez más importantes en la toma de decisiones de proyectos, por lo cual el ofrecimiento de servicios en la nube les permite disminuir los costos de la compañía, como se pudo observar en el análisis de TCO, lo cual, adicionalmente, también se refleja en tiempos más cortos del Retorno de la Inversión (ROI). 


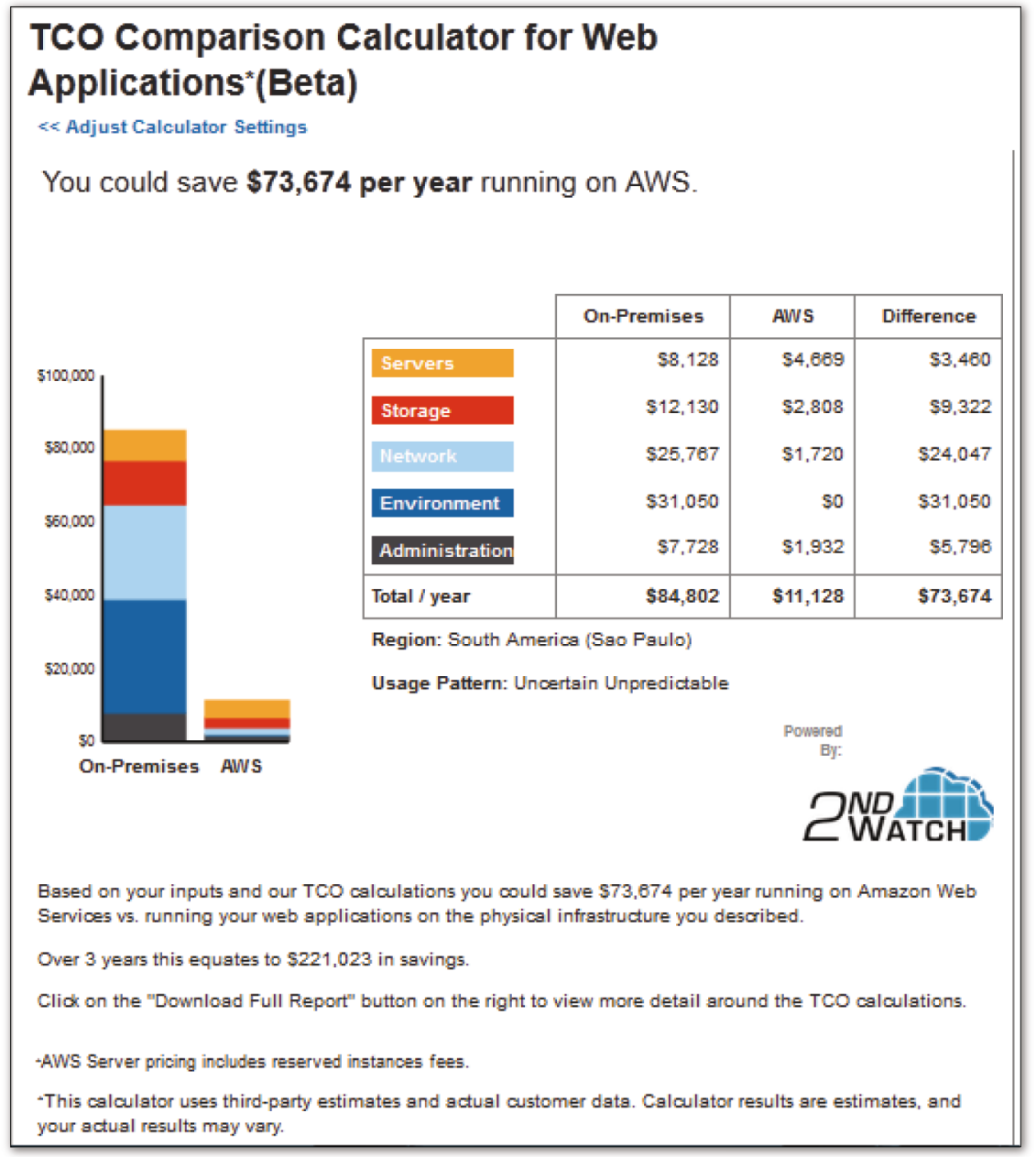

Fig. 5. AMAZON - TCO Comparison Calculator for Web Applications

\section{Trabajos futuros}

El Estado colombiano debe generar una legislación para la contratación de servicios en la nube que incluya las recomendaciones descritas en este documento; de esta manera podrá haber una adopción segura y efectiva de Cloud Computing para reducir los costos y mejorar los servicios, tal y como lo hace actualmente el NIST del Gobierno de los Estados Unidos, o la Agencia Española de Protección de Datos.

Específicamente para el caso de Colombia, se detectó que hay una omisión respecto al nivel de protección de datos y las políticas de transferencia internacional de datos, la cual permite, en este momento, que los proveedores de servicios en la nube no se vean obligados a brindar garantías sobre el manejo de la información de los clientes en caso de que realicen transferencias internacionales de los datos. Sin esta protección, cuando la información de las empresas estatales se encuentre fuera de Colombia, las leyes que se van a aplicar sobre el manejo de ella serán las del país tercero, lo cual genera grandes riesgos sobre la confidencialidad y protección de la información, ya que no tiene garantías jurídicas adecuadas.

La computación en nube se encuentra todavía en una etapa de rápido despliegue, y las normas son cruciales para una mayor adopción por el mercado. Las normas son fundamentales para garantizar la igualdad de condiciones en el mercado global. 


\section{Conclusiones}

El papel de los gerentes de finanzas, o CFO, en las empresas se ha convertido en un punto clave para la toma de decisiones en ellas. Con base en el análisis de Costo Total de Propiedad realizado se obtiene una justificación sólida para demostrar la viabilidad y las ventajas económicas que trae a la compañía la migración a una compra de soluciones de tecnología bajo la modalidad de computación en la nube, la cual, por su esquema de servicios, permite a las compañías enfocar sus esfuerzos e inversiones en el Core del negocio para generar innovación que les permite posicionarse en el mercado y así no gastar su capital en la operación del día tras día, que genera altos costos y retrasa el crecimiento de las empresas.

La adopción de la computación en la nube es probable que tenga otras consecuencias para el departamento de TI. A medida que más servicios se obtienen de los proveedores de la nube, la necesidad de funciones dentro del departamento de TI que sirvan para administrar, supervisar y mantener la infraestructura de TI se verá disminuida considerablemente o incluso eliminada. Bajo el Cloud Computing, el departamento de TI debe aumentar su foco en el valor agregado a los servicios entrantes basados en la nube. La misión del departamento de TI será la transición a la determinación de los medios por los que el personal de TI puede asegurar que las mejoras a los servicios basados en la nube se ajustan a las necesidades actuales y futuras de las unidades que los consumen. El impacto de estos cambios puede ser objeto de futuras investigaciones.

Los Acuerdos de Nivel de Servicio (ANS) son elementos clave que se deben especificar en los contratos de prestación de servicios en la nube para garantizar el correcto manejo de los datos, y, así mismo, son requisitos especiales de seguridad, capacidad, continuidad, disponibilidad, confidencialidad e integridad del servicio, incluyendo exigencias en la gestión y monitoreo del servicio; además, el proveedor de servicios debe entregar a sus clientes reportes de la prestación y operación del servicio en los cuales pueda relacionar los ANS incluidos en el contrato; estos reportes son muy importantes, ya que les permiten a los clientes ver si su proveedor de servicios en la nube está cumpliendo o no con los ANS incluidos en el contrato. En ciertos casos, la complejidad de los servicios contratados puede aconsejar la designación de un responsable del contrato, con el fin de asegurar la correcta realización de la prestación pactada.

\section{REFERENCIAS}

[1] "Computación en la nube". En Wikipedia, la enciclopedia libre. Recuperado el 17 de junio de 2014 de: http://es.wikipedia.org/wiki/ Computaci\%C3\%B3n_en_la_nube.

[2] SDI, ServiceDesk Institute. ITILV3 SERVICE MANAGEMENT FOUNDATION. ITIL Foundation V3 Rel 1.2. 2009.

[3] Gómez, Inc. (2006). Service Level Agreements and Management: Putting RealWorld Management in SLM and Next Steps in SLA. White Paper.

[4] O. M. Gómez, Gestión de los riesgos del proyecto basado en los estándares del PMI®. San José, Costa Rica. 2012.

[5] V.J. Zapata, "Valor Monetario Esperado". Gerencia de Proyectos. Recuperado el 7 de Junio de 2014, de: http://ceresegp.blogspot. com/2011/04/valor-monetario-esperado.html.

[6] L. Mora, Cálculo del TCO y del ROI para proyectos informáticos. Apuntes de computación. Recuperado el 6 de junio de 2014, de: http://apuntescomputacion.wordpress. com/2008/08/16/calculo-del-costo-total-de-propiedadtco/.

[7] España, Agencia Española de Protección de Datos. Guía para clientes que contraten servicios de Cloud Computing. Recuperado el 7 de junio de 2014, de www.agpd.es.

[8] IDC, International Data Corporation. (2012). Cloud Research. Framingham, MA. Recuperado el 7 de junio de 2014, de www. idc.com/prodserv/idc_cloud.jsp.

[9] Colombia. "Manual para la implementación de la estrategia de Gobierno en línea en las 
entidades del orden nacional de la República de Colombia". MinTIC -Ministerio de las Tecnologías de la Información y las Comunicaciones-. 2012.

[10] A. Adamov \& M. Erguvan, The truth about cloud computing as new paradigm in IT. 2009. International Conference on Application of Information and Communication Technologies, 9-11. doi: 10.1109/ICAICT.2009.5372585. 2009

[12] M. Kelly, "The enhanced Telecom Operation Map (eTOM). Update on ongoing work". Presented in: TeleManagement World, Dallas. 2003.

[13] P. Mell \& T. Grance, The NIST definition of cloud computing. Special Publication 800145, National Institute of Standards and
Technology, U.S. Department of Commerce. January 2011.

[14] P. Patel, A. Ranabahu \& A. Sheth, Service Level Agreement in cloud computing. Cloud Workshops at OOPSLA. 2009.

[15] R. Tisnovsky, "Risks Versus Value in Outsourced Cloud Computing". Financial Executive, 26, 64-66. 2010.

[16] United States, NIST -National Institute of Standards and Technology-. Cloud Computing Technology Roadmap Volume. Recuperado el 7 de junio de 2014, de: http:// www.nist.gov/itl/cloud/upload/SP_500_293_ volumeI-2.pdf.

[17] J. Yang \& Z. Chen, Cloud Computing Research and Security Issues. IEEE, Computational Intelligence. 2010. 